\title{
Canadian
}

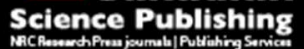

Canadian Journal of Physics

Revue canadienne de physique

\section{Photoionization of the francium 7P3/2 state}

\begin{tabular}{|r|l|}
\hline Journal: & Canadian Journal of Physics \\
\hline Manuscript ID & cjp-2016-0311.R2 \\
\hline Manuscript Type: & Article \\
\hline Date Submitted by the Author: & 27-Aug-2016 \\
\hline Complete List of Authors: & $\begin{array}{l}\text { Collister, Robert; TRIUMF, ; University of Manitoba, Physics \& Astronomy } \\
\text { Zhang, Jiehang; University of Maryland at College Park } \\
\text { Tandecki, Michael; TRIUMF } \\
\text { Aubin, Seth; College of William and Mary, Physics } \\
\text { Gomez, Eduardo; Universitat Autonoma de San Luis Potosi, Fisica } \\
\text { Gwinner, Gerald; University of Manitoba, Physics and Astronomy } \\
\text { Orozco, Luis; University of Maryland } \\
\text { Pearson, Matthew; TRIUMF } \\
\text { Behr, John; TRIUMF }\end{array}$ \\
\hline Keyword: & photoionization, alkali atoms, magneto-optical trap, francium, excited state \\
\hline
\end{tabular}




\title{
Photoionization of the francium $7 P_{3 / 2}$ state
}

\author{
R. Collister, ${ }^{1, *}$ J. Zhang, ${ }^{2}$ M. Tandecki, ${ }^{3}$ S. Aubin, ${ }^{4}$ E. Gomez,${ }^{5}$ \\ G. Gwinner, ${ }^{1}$ L. A. Orozco, ${ }^{2}$ M.R. Pearson, ${ }^{3}$ and J.A. Behr ${ }^{3}$ \\ ${ }^{1}$ Department of Physics and Astronomy, University of Manitoba, Winnipeg, MB R3T 2N2, Canada \\ ${ }^{2}$ JQI, Department of Physics and NIST, University of Maryland, College Park, MD 20742, U.S.A. \\ ${ }^{3}$ TRIUMF, Vancouver, BC V6T 2A3, Canada \\ ${ }^{4}$ Department of Physics, College of William and Mary, Williamsburg, VA 2319, U.S.A. \\ ${ }^{5}$ Instituto de Física, Universidad Autónoma de San Luis Potosí, San Luis Potosi 78290, Mexico
}

We have measured the non-resonant photoionization cross-section of the $7 P_{3 / 2}$ state of francium for $442 \mathrm{~nm}$ light to be $20.8 \pm 7.1 \mathrm{Mb}$. Atoms were irradiated in a magneto-optical trap, and we deduce the photoionization rate from the change in trap lifetime. The result is consistent with a simple extrapolation of known cross-sections for other alkali atoms.

\footnotetext{
* Corresponding author: rcollister@triumf.ca
} 


\section{INTRODUCTION}

The near-threshold photoionization of atoms is an important process to consider as a loss mechanism in two-species magneto-optical traps [1]. It is also of indirect importance in atomic parity violation experiments, either as a loss mechanism $[2,3]$ or as a detection scheme [4]. A detailed calculation has been done of the francium $7 S$ ground state photoionization cross-section [5], but we are unaware of calculations for the $P$ states. However, photoionization from the $P$ states has been explored in good detail in the other alkali atoms [6]. High precision, absolute measurements of photoionization cross-sections are difficult to achieve for several reasons; most notable are the requirements for the knowledge of the absolute number of atoms as well as the absolute photon flux [7, 8].

The use of a magneto-optical trap (MOT) allows for alternative techniques that are not possible to implement in a beam experiment [9]. The MOT lifetime is determined by a combination of effects, each of which removes atoms from the trap. Typically, for long-lived traps, the atom loss is dominated by collisions with background gas and hence determined by the vacuum pressure. If the trapped species is unstable, the radioactive lifetime must also be considered, and the total loss rate is the sum of all mechanisms, producing an exponential decay of trapped atoms. This is the case for francium, which has no stable isotopes. In a sufficiently dilute MOT where two-body losses are negligible, the number of atoms in the trap decays as:

$$
N(t)=N_{0} e^{-t\left(1 / \tau_{1}+1 / \tau_{2}\right)}=N_{0} e^{-R t},
$$

with $N(t)$ atoms at time $t$, and $N_{0}$ atoms are present at $t=0$. Then $\tau_{i}$ is the lifetime determined from a single trap loss mechanism, such as collisions with background gas or radioactive decay, and $R$ is the combined loss rate from all of the mechanisms.

The addition of photoionization introduces another loss mechanism; ions do not experience the resonant radiative forces of the MOT and are thereby lost. With the only difference being the presence of the ionizing light, the change in the trap lifetime is attributed to photoionization and we use this to calculate the cross-section [9]. Thus, the photoionization rate $R_{\mathrm{PI}}$ is determined by:

$$
R_{\mathrm{PI}}=\frac{1}{\tau_{\mathrm{on}}}-\frac{1}{\tau_{\mathrm{off}}}
$$

where $\tau_{\mathrm{on}(\mathrm{off})}$ is the trap lifetime with the photoionizing light on(off). If a single photon has sufficient energy to ionize from a particular level in the MOT, we can identify the photoionization rate as coming from that electronic state, neglecting higher order effects such as two-photon excitations or photoassociation, which would also remove atoms from the trap but require higher laser intensities. Once the photoionization rate is known, the cross-section from that excited state can be calulated from [9]:

$$
\sigma=\frac{R_{\mathrm{PI}}}{n_{e} \Phi}
$$

where $n_{e}$ is the fraction of trapped atoms in the excited state from which photoionization occurs and $\Phi=I / h \nu$ is the photon flux (photons $\mathrm{s}^{-1} \mathrm{~cm}^{-2}$ ) of the ionizing light of intensity $I\left(\mathrm{~W} / \mathrm{cm}^{2}\right)$ at frequency $\nu$. The excited state fraction is determined from the conditions of the MOT and the properties of the trapped atoms. This equation is valid where the photoionizing rate is linear with the applied light, i.e. we are not saturating the process with high intensities of light. Ref. [7] observes saturation in Na and K atoms with pulsed intensities greater than $100 \mathrm{MW} / \mathrm{cm}^{2}$, whereas we operate below $1 \mathrm{~W} / \mathrm{cm}^{2}$, placing us well below saturation.

This technique does not need to know the absolute number of atoms interacting with the ionizing light; instead it requires knowledge of the fraction of atoms involved in the interaction. This quantity corresponds to the fraction of atoms in an excited state that can be photoionized. Some measurements use this to their advantage and saturate the transition [9]. We calculate our excited state fraction from the parameters of the trap. Furthermore, this technique is a fluorescence measurement and does not require any ion detection. It can be carried out directly in the MOT.

\section{EXPERIMENTAL METHOD}

Our francium experiments [10], with no stable isotopes, require the limited online isotope production beamtimes available at TRIUmF. This simple experiment was designed to produce a measurement accurate enough to plan future atomic parity violation experiments [3]. Francium ions were delivered to our facilty where they were captured in a thin yttrium foil neutralizer, which was then rotated into position and heated to release neutral francium into our trapping volume; this loaded the trap on a 20 s cycle. After 1 second of heating, the yttrium foil was rotated back to 
the catching position. It was irradiated in the heating position by the $442 \mathrm{~nm}$ beam, but it was not exposed to the photoionizing light during measurements of the MOT lifetime. The measurement probed a sample of ${ }^{221} \mathrm{Fr}$ contained within the MOT, which has a radioactive half-life of 4.8 minutes. This was sufficiently long that we do not need to account for nuclear decay during our lifetime measurements. We measured the change in the trap lifetime when non-resonant $442 \mathrm{~nm}$ laser light was applied compared to the lifetime when that light was blocked. Illumination of the trap with photoionization light occured every other cycle. Our result for the total cross-section is not sensitive to the $s$ - and $d$-waves of the outgoing electron, as measured in other experiments [6], as the quadrupole magnetic field and alternately polarized six beams of the MOT cause any polarization in the atom cloud to be small.

The $442 \pm 2 \mathrm{~nm}$ light was from an unregulated Laserglow Polaris-200 battery powered diode laser calibrated by a simple grating spectrometer, which was in turn calibrated using the $436 \mathrm{~nm}$ mercury line. The light was transported through an optical fiber and collimated to a beam with $1 / e^{2}$ diameter $10 \mathrm{~mm}$, whose spatial profile was measured using a calibrated iris and photodiode. To determine the alignment of the photoionization beam with the MOT, rubidium atoms were trapped after the francium measurement in the same location, and light resonant with the 780 $\mathrm{nm}$ rubidium D2 line was sent through the fiber. The calibrated aperture was adjusted until the probe light destroyed the MOT. This measured how off-center the beam position was, which we found to be 0.75 to $1.5 \mathrm{~mm}$, and, along with a power measurement, we determined the light intensity incident on the atom cloud to be $41 \pm 6 \mathrm{~mW} / \mathrm{cm}^{2}$, corresponding to a photon flux of $9.1 \pm 1.3 \times 10^{16} \mathrm{~s}^{-1} \mathrm{~cm}^{-2}$.

The MOT fluorescence was measured by a CCD camera, and the recorded integrated signal was analyzed to determine the trap lifetime. An absolute calibration of the camera count-to-atoms was not required as we only needed the change in fluoresence to fit the lifetime. As we were not saturating the CCD chip, the camera response was linear to the fluorescence, and we could determine the trap lifetime by fitting simple exponentials.

\section{RESULTS}

\section{A. Excited state fraction}

The excited state fraction is determined from the conditions of the MOT and the isotope trapped, as we were unable to saturate the transition as in [9].

The $442 \mathrm{~nm}$ laser light is of sufficient energy to photoionize not only the $7 P_{3 / 2}$ level but the $7 P_{1 / 2}$ level as well. We find that five levels are needed to accurately represent the system and determine their populations by a five-level rate equation. The levels involved are those at or near-resonance with either the trapping or repumping laser, as shown in Fig. 1. Also included is the $|F=3\rangle 7 P_{3 / 2}$ level with natural linewidth $7.57 \mathrm{MHz}$ [11] separated from the $|F=4\rangle$ level by $58 \mathrm{MHz}$ [12], which is close enough to the trapping laser frequency such that its finite lineshape is sufficient to cause a non-negligible population. We approximate the light as isotropically polarized, as the magnetic quadrupole field of the MOT scrambles the quantization axis of the atoms in the trapping volume. Other excited states are not included in the rate equation; the relatively large hyperfine splittings make their steady-state populations in the trap negligible $\left(<10^{-4}\right)$.

The trapping laser has an intensity of $22 \pm 5 \mathrm{~mW} / \mathrm{cm}^{2}$ with detuning $12.0 \pm 3.7 \mathrm{MHz}$ below the $|F=3\rangle \rightarrow\left|F^{\prime}=4\right\rangle$ $718 \mathrm{~nm}$ cycling transition, coupling also to the $\left|F^{\prime}=3\right\rangle$ state $58 \mathrm{MHz}$ below the $\left|F^{\prime}=4\right\rangle$ state. The repumping laser is tuned to within $3 \mathrm{MHz}$ of the $|F=2\rangle \rightarrow\left|F^{\prime}=3\right\rangle 817 \mathrm{~nm}$ transition with intensity $1.4 \pm 0.5 \mathrm{~mW} / \mathrm{cm}^{2}$. Combining the laser parameters with the state lifetimes, $21.02 \mathrm{~ns}$ and $29.45 \mathrm{~ns}$ for the $7 P_{3 / 2}$ and $7 P_{1 / 2}$ states respectively [11], provides all that is needed to calculate the excited state fractions. Solving analytically for the steady-state populations produces the total excited state fraction $n_{e}($ full $)=0.12 \pm 0.04$. Given careful studies in MOTs of effective saturation intensities from dynamic and other effects (see e.g. [13]), we have varied the theoretical saturation intensity by $30 \%$ and found this does not increase our uncertainty given the other significant systematics in our 5-level system.

The contribution from the $7 P_{1 / 2}$ state is $n_{e}\left(7 P_{1 / 2}\right)=0.0031$. Measurements in cesium [8] show that the difference in the cross-sections for the first excited $P_{1 / 2}$ and $P_{3 / 2}$ states is small, once the photon energy has exceeded the threshold for photoionization for both states. We assume that this is also true for francium. Thus we neglect this small difference compared to other sources of error and include the contribution of the $7 P_{1 / 2}$ state directly in the above total excited state fraction.

\section{B. Trap lifetimes}

We fit the peaks in our data to simple exponentials on a fixed, flat background, as shown in Fig. 2. The background level is determined by observing the data where the trap was void of atoms. Additionally, we check that this background level is consistent with the data between short lifetime peaks where most atoms have been lost. The 


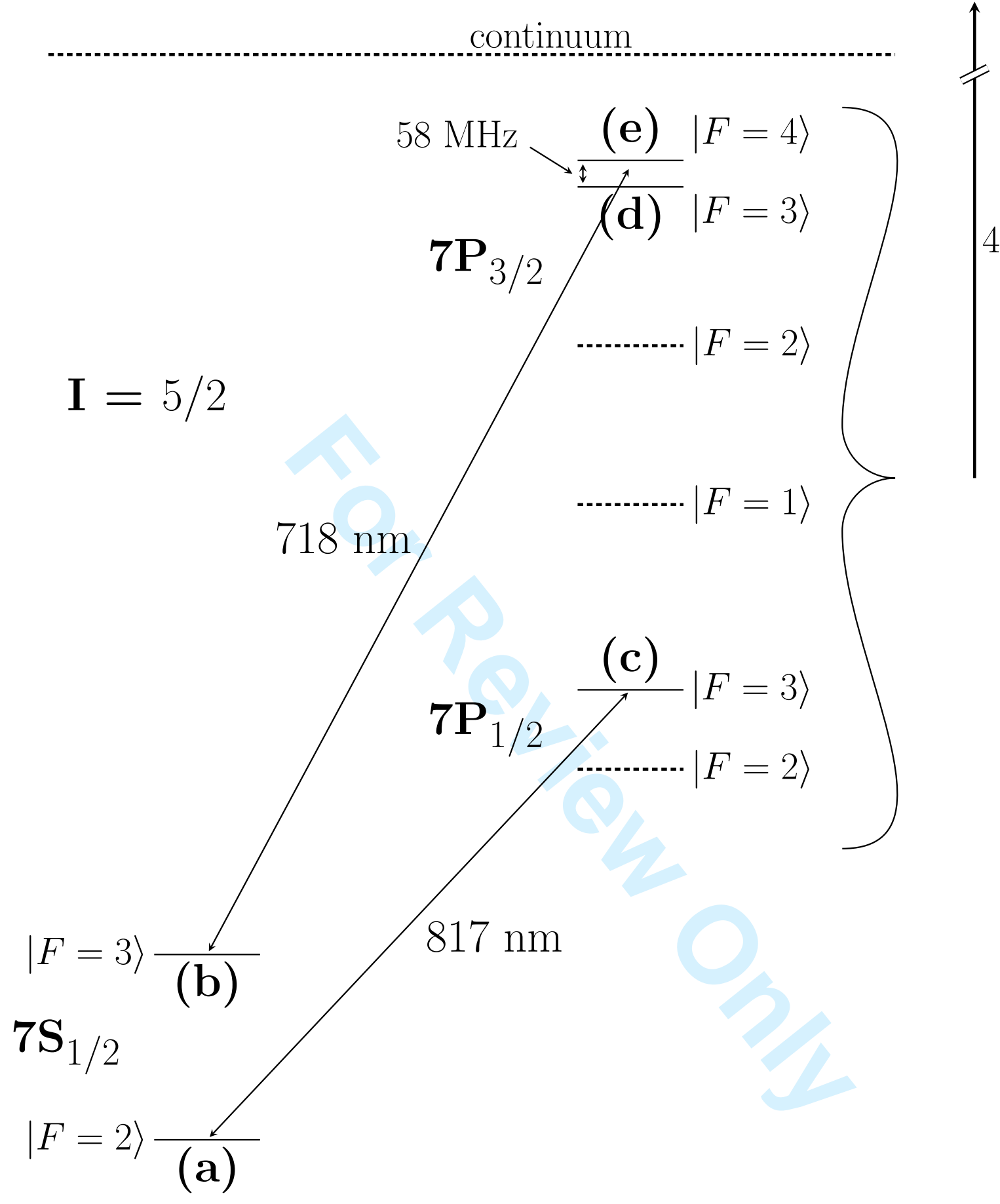

FIG. 1. ${ }^{221}$ Fr energy levels involved in the rate equation. The trap (718 nm) and repump (817 nm) lasers couple the solid-lined levels (a) - (e) such that in the steady-state, those levels have non-zero population. Dash-lined levels are sufficiently detuned from the lasers to not be involved. The $442 \mathrm{~nm}$ light is of sufficient energy to photoionize all $7 P$ states with a single photon transition. Hyperfine splittings obtained from [12].

uncertainty in the fits is dominated by significant scatter in the signal, mostly attributable to power fluctuations in the delivered trap light since it also appears when there are no atoms in the trap. We include the variation from these fluctuations in the quoted uncertainty. The fit results are shown in Table I along with their weighted averages. Since the chi-squared per degree of freedom $\chi^{2} / \operatorname{dof}=\sum\left(w_{i}\left(\bar{\tau}-\tau_{i}\right)^{2}\right) /$ dof $=1.73$ and 2.04 (confidence level $14 \%$ and $8 \%$ ), for light-on and -off respectively, we inflate the uncertainties of the weighted averages by $\sqrt{\chi^{2} / \text { dof. }}$. 


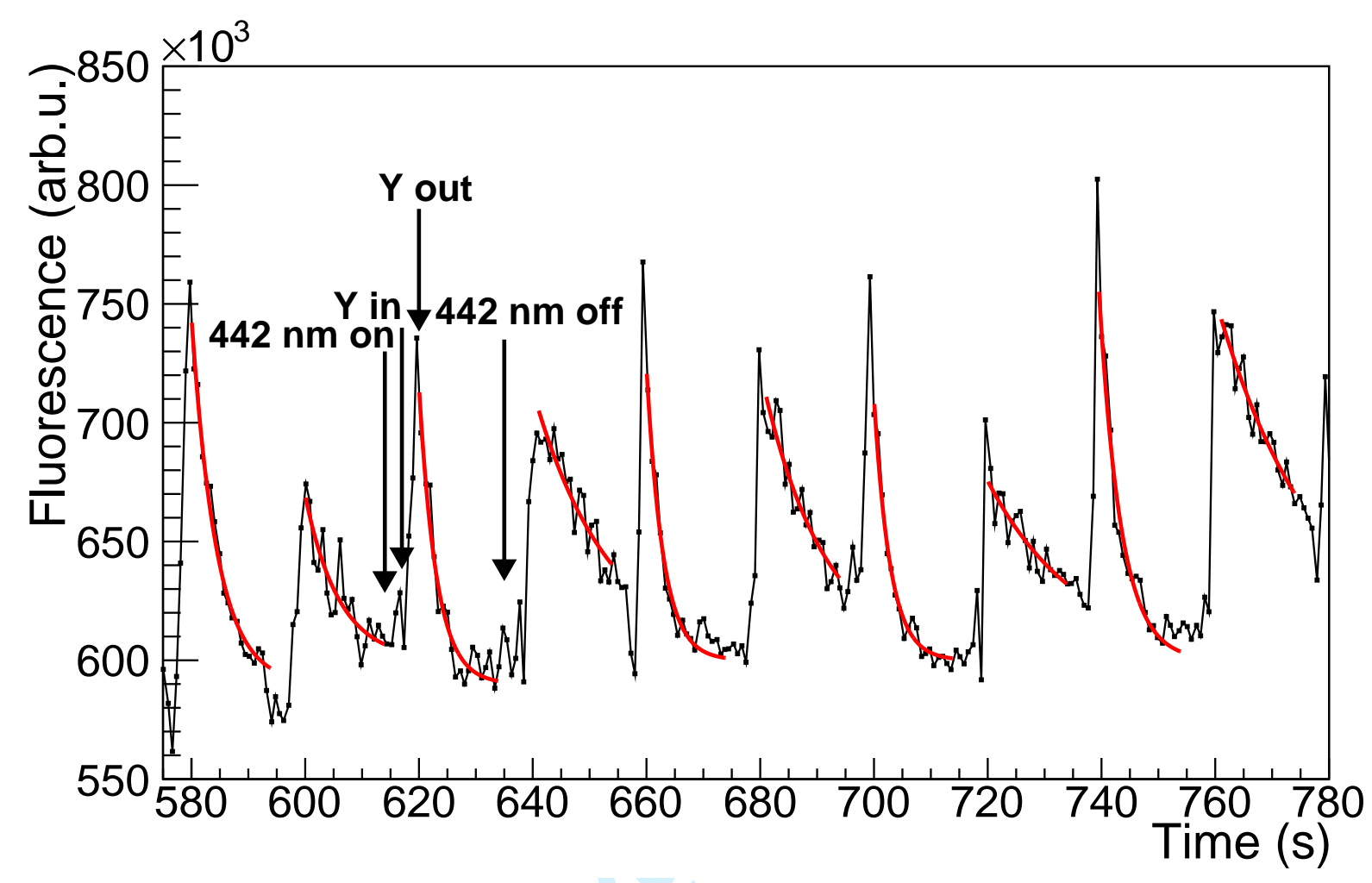

FIG. 2. Data showing peaks of the trap loading cycle with alternating $442 \mathrm{~nm}$ light on/off. The times for the Y foil rotating in/out as well as the times where the $442 \mathrm{~nm}$ light is turned on/off are indicated with arrows for one cycle. Red lines represent the fits of exponentials to the peaks.

\section{Constraints on photodesorption and photoassisted dimer formation}

There is an increase of fluorescence signal when the $442 \mathrm{~nm}$ light is applied. Our beam only irradiates the yttrium catcher foil and 1/30 of the vapour cell. Since we heat the foil enough to release approximately $10 \%$ of the captured francium per shot, and the foil is moved out of the beam after 1 second, we attribute the additional atoms to photodesorption of francium from the yttrium, as observed at INFN-LNL [14].

Light-assisted desorption (LIAD) of rubidium is used to load MOTs from uncoated pyrex cells in geometries optimized for that purpose $[15,16]$. LIAD could in principle introduce a loss rate for our trapped francium atoms by collisions with added $\mathrm{Rb}$ atoms, since we have a small amount of residual $\mathrm{Rb}$ in our geometry from offline tests of our trap [10]. We conservatively constrain this possibility by considering the trap loss rates observed in Ref. [16], scaling by the 1/30 fraction of our cell irradiated with the same intensity, and assuming the possible fraction of bad dryfilm is less than $10 \%$ [10]. Thus we estimate considerably less than a $0.0002 s^{-1}$ trap loss rate in our system from photodesorption of $\mathrm{Rb}$ with the $442 \mathrm{~nm}$ light. We conclude that there are additional francium atoms in the MOT from photodesorption from the yttrium foil by the $442 \mathrm{~nm}$ light, but the effect on the trap lifetime, and hence the photoionization rate, from photodesorption is negligible within our precision.

In principle, the $442 \mathrm{~nm}$ light could allow photoassisted dimer formation, since it is to the red of the $7 S$ to $8 P$ transitions, and the molecular formation rate would have to be differentiated from the photoionization rate. However, we can constrain the formation rate during our photoionization measurement to be negligible using literature data and theory. Ref. [17] saturated a Cs $6 \mathrm{~S} \rightarrow 6 \mathrm{P}_{3 / 2}$ photoassisted molecular resonance with intensity $I=55 \mathrm{~W} / \mathrm{cm}^{2}$, measuring a loss rate $2.5 \mathrm{~s}^{-1}$ in a MOT with atom density $n=10^{11} \mathrm{~cm}^{-3}$ and temperature $T=140 \mu \mathrm{K}$. Their saturated rate agreed with the theoretical on-resonance maximum $n v_{\text {rel }} \pi / k^{2}$, where $v_{r e l}$ is the relative velocity and $k$ is the atom momentum [18]. Calculations including relativistic corrections predict that francium should have similar photoassociation processes as cesium [19]. We scale the measured maximum rate of Ref. [17] by our much smaller parameters $n=10^{8} \mathrm{~cm}^{-3}$ and $I=41 \pm 6 \mathrm{~mW} / \mathrm{cm}^{2}$, and we assume $T \geq 10 \mu \mathrm{K}$ to get a maximal rate estimate. Noting that our light is to the blue of the $7 \mathrm{~S} \rightarrow 7 \mathrm{P}$ transitions, forbidding formation of photoassisted molecules from them, we also scale by the weaker strength of the 436 and $426 \mathrm{~nm} 7 \mathrm{~S} \rightarrow 8 \mathrm{P}$ transitions [20]. The resulting upper limit for 
photoassisted dimer formation in our measurement is $\sim 3 \times 10^{-6} \mathrm{~s}^{-1}$, which we neglect.

\section{Experimental results}

The average lifetimes, shown in Table I, are converted to loss rates, then subtracted to extract the loss rate due to photoionization according to equation 2 . We find $R_{\mathrm{PI}}=0.230 \pm 0.028 \mathrm{~s}^{-1}$.

TABLE I. Exponential lifetimes obtained by fitting peaks in Fig. 2. The weighted averages have had their errors inflated by their respective $\sqrt{\chi^{2} / \text { dof. }}$

\begin{tabular}{cc}
\hline$* \tau_{\text {on }}(\mathrm{s})$ & $\tau_{\text {off }}(\mathrm{s})$ \\
\hline $3.6 \pm 0.4$ & $7.7 \pm 2.8$ \\
$3.4 \pm 0.5$ & $15.8 \pm 2.9$ \\
$2.7 \pm 0.3$ & $15.4 \pm 2.8$ \\
$4.0 \pm 0.5$ & $12.8 \pm 1.7$ \\
$3.6 \pm 0.5$ & $19.5 \pm 3.7$ \\
\hline * average: $\tau_{\text {on }}=3.29 \pm 0.24 \mathrm{~s}$ average: & $\tau_{\text {off }}=13.5 \pm 1.6 \mathrm{~s}$
\end{tabular}

Combining the measured photon flux and photoionization rate with the calculated excited state fraction produces a photoionization cross-section of $2.08 \pm 0.71 \times 10^{-17} \mathrm{~cm}^{2}$ or $20.8 \pm 7.1 \mathrm{Mb}$ for ${ }^{221} \mathrm{Fr}$ by $442 \mathrm{~nm}$ light.

\section{Comparison to other alkalis}

A review of photoionization cross-sections of excited alkali atoms can be found in [6], including experimental values for $\mathrm{Na}[7,21], \mathrm{K}[6], \mathrm{Rb}$ [9] and $\mathrm{Cs}[8,22]$. It also contains theoretical treatments of the alkali elements up to cesium, showing the photoionization cross-sections for different photoelectron energies, for different calculations. Additionally, photoionization cross-section measurements have been performed for many other excited states of the aklalis, e.g. [2325] for $\mathrm{Li},[23,26]$ for $\mathrm{Na}$, and [27-30] for $\mathrm{K}$. For our $442 \mathrm{~nm}$ light photoionizing the $7 \mathrm{P}_{3 / 2}$ state, the photoelectron energy is $E_{e}=0.46 \mathrm{eV}$. We can obtain experimental cross-sections near our photoelectron energy for the other alkalis and compare with our result for francium. Published numbers for the first excited $n P_{3 / 2}$ state from the other alkali atoms have been interpolated using theory [6] to project cross-sections closer to $0.46 \mathrm{eV}$, and the cross-sections from the review [6] are plotted in Fig. 3.

\section{CONCLUSION}

We have measured the non-resonant photoionization cross section of the $7 P_{3 / 2}$ state of francium for $442 \mathrm{~nm}$ light to be $20.8 \pm 7.1 \mathrm{Mb}$. We irradiated atoms in a magneto-optical trap and measured the change in trap lifetime to deduce the photoionization rate. The result is in line with the general trend exhibited by photoionization cross-sections for the other alkali atoms. It is of sufficient precision to estimate the magnitude of the loss rate from photoionization in a francium trap for an atomic parity violation experiment [3].

\section{ACKNOWLEDGMENTS}

The authors would like to thank the ISAC staff at TRIUMF for developing the Fr beam. This work is supported by Natural Sciences and Engineering Research Council of Canada, United States Department of Energy and National Science Foundation, and Consejo Nacional de Ciencia Y Tecnologia from Mexico. R.C. acknowledges support from a University of Manitoba Graduate Fellowship. TRIUMF receives federal funding via a contribution agreement with the National Research Council of Canada.

[1] T. Aoki, Y. Yamanaka, M. Takeuchi, Y. Torii, and Y. Sakemi. Phys. Rev. A 87, 063426 (2013). 


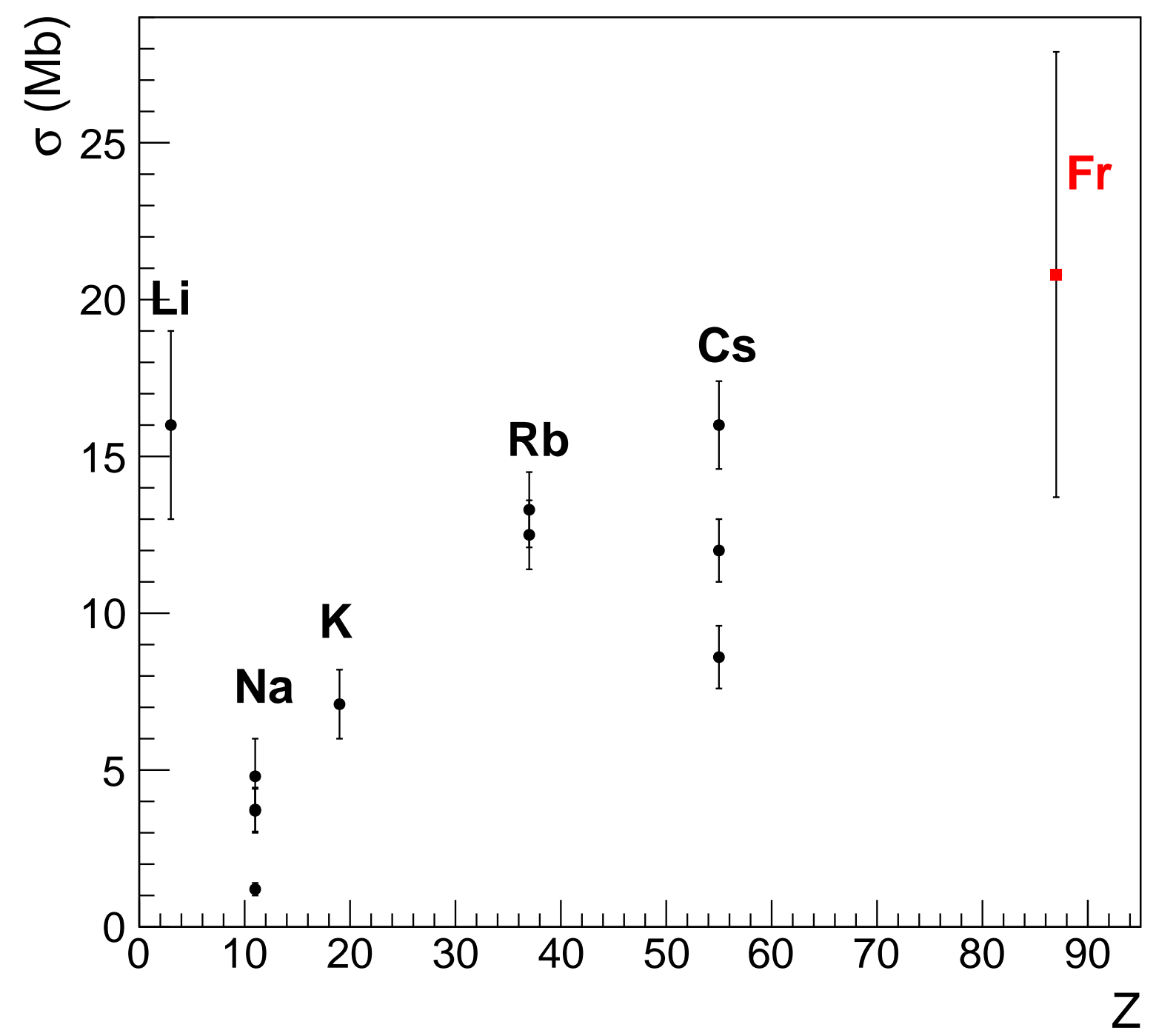

FIG. 3. Comparison of experimental photoionization cross-sections of the first excited $n P_{3 / 2}$ state of the alkali atoms for photoelectron energies at $0.46 \mathrm{eV}$, projected from measurements near $0.46 \mathrm{eV}$ and theory interpolation. Our francium $(\mathrm{Z}=87)$ measurement at $0.46 \mathrm{eV}$ follows the general trend set by the lighter alkalis [6] except for lithium [23].

[2] D. Vieira, C. Wieman, et al. Los Alamos Meson Physics Facility Research Proposal 1303 (1992), section VI.

[3] J.A. Behr, and G. Gwinner. J. Phys. G 36, 033101 (2009), section 3.4.4

[4] E.D. Commins. Phys. Scr. 36, 468 (1987).

[5] A. Derevianko, W.R. Johnson, and H.R. Sadeghpour. Phys. Rev. A 61, 022506 (2000).

[6] I.D. Petrov, V.L. Sukhorukov, E. Leber, and H. Hotop. Eur. Phys. J. D 10, 53 (2000).

[7] C.E. Burkhardt, J.L. Libbert, J. Xu, J.J. Leventhal, and J.D. Kelley. Phys. Rev. A 38, 5949 (1988).

[8] K.J. Nygaard, R.E. Hebner Jr., J.D. Jones, and R.J. Corbin. Phys. Rev. A 12, 1440 (1975).

[9] T.P. Dinneen, C.D. Wallace, K.-Y.N. Tan, P.L. and Gould. Opt. Lett. 17, 1706 (1992).

[10] M. Tandecki, J. Zhang, R. Collister, S. Aubin, J.A. Behr, E. Gomez, G. Gwinner, L.A. Orozco, and M.R. Pearson. J. Instrum. 8, P12006 (2013).

[11] J.E. Simsarian, L.A. Orozco, G.D. Sprouse, and W.Z. Zhao. Phys. Rev. A 57, 2448 (1998).

[12] Z.-T. Lu, K.L. Corwin, K.R. Vogel, C.E. Wieman, T.P. Dinneen, J. Maddi, and H. Gould. Phys. Rev. Lett. 79, 994 (1997).

[13] G. Veshapidze, J.-Y. Bang, C.W. Fehrenbach, H. Nguyen, and B.D. DePaola. Phys. Rev. A 91, 053423 (2015).

[14] V. Coppolaro, et al. J. Chem. Phys. 141, 134201 (2014). 
[15] M.K. Ivory, A.R. Ziltz, C.T. Fancher, A.J. Pyle, A. Sensharma, B. Chase, J.P. Field, A. Garcia, D. Jervis, and S. Aubin, Rev. Sci. Instrum. 85, 043102 (2014).

[16] M.H.T. Extavour, L.J. LeBlanc, T. Schumm, B. Cieslak, S. Myrskog, A. Stummer, S. Aubin, and J.H. Thywissen. AIP Conf. Proc. 869, 241 (2006).

[17] C. Drag, et al. IEEE J. Quantum Electron 36, 1378 (2000).

[18] K.M. Jones, E. Tlesinga, P.D. Lett, and P.S. Jullenne. Rev. Mod. Phys. 78, 483 (2006).

[19] M. Aymar, O. Dulieu, and F. Spiegelman. J. Phys. B 39, S905 (2006).

[20] J.E. Sansonetti. J. Phys. Chem. Ref. Data 36, 497 (2007).

[21] J.M. Preses, C.E. Burkhardt, R.L. Corey, D.L. Earsom, T.L. Daulton, W.P. Garver, J.J. Leventhal, A.Z. Msezane, and S.T. Manson. Phys. Rev. A 32, 1264 (1985).

[22] O. Maragò, D. Ciampini, F. Fuso, E. Arimondo, C. Gabbanini, and S.T. Manson. Phys. Rev. A 57, R4110 (1998).

[23] V. Wippel, C. Binder, W. Huber, L. Windholz, M. Allegrini, F. Fuso, and E. Arimondo. Eur. Phys. J. D 17, 285 (2001).

[24] M. Saleem, S. Hussain, M. Rafiq, and M.A. Baig. J. Phys. B 39, 5025 (2006).

[25] M. Saleem, S. Hussain, and M.A. Baig. Phys. Rev. A 77, 062506 (2008).

[26] N. Amin, S. Mahmood, M. Anwar-ul-Haq, M. Riaz, and M.A. Baig. Eur. Phys. J. D 37, 23 (2006).

[27] N. Amin, S. Mahmood, S.U. Haq, M.A. Kalyar, M. Rafiq, and M.A. Baig. J. Quant. Spectrosc. Radiat. Transfer 109, 863 (2008).

[28] A. Yar, R. Ali, and M.A. Baig. Phys. Rev. A 87, 045401 (2013).

[29] A. Yar, R. Ali, and M.A. Baig. Phys. Rev. A 88, 033405 (2013).

[30] M.A. Kalyar, A. Yar, J. Iqbal, R. Ali, and M.A. Baig. Opt. Laser Technol. 77, 72 (2016). 